Published in final edited form as:

Lab Chip. 2019 February 12; 19(4): 665-673. doi:10.1039/c81c01267a.

\title{
Large-Scale Production of Compound Bubbles Using Parallelized Microfluidics for Efficient Extraction of Metal Ions
}

\author{
Heon-Ho Jeong ${ }^{a, b}$, Zhuo Chen ${ }^{a, d}$, Sagar Yadavalic ${ }^{c}$ Jianhong $\mathbf{X u}^{\mathrm{d}}$, David Issadore ${ }^{\mathrm{a}, \mathrm{c}, \mathrm{e}}$, and \\ Daeyeon Lee ${ }^{a}$ \\ aDepartment of Chemical and Biomolecular Engineering, University of Pennsylvania, \\ Philadelphia, Pennsylvania 19104, United States \\ ${ }^{b}$ Department of Chemical and Biomolecular Engineering, Chonnam National University, Yeosu, \\ Jeonnam 59626, Republic of Korea \\ 'Department of Bioengineering, University of Pennsylvania, Philadelphia, Pennsylvania 19104, \\ United States \\ dThe State Key Laboratory of Chemical Engineering, Department of Chemical Engineering, \\ Tsinghua University, Beijing 100084, China \\ eElectrical and Systems Engineering, School of Engineering and Applied Sciences, University of \\ Pennsylvania, Philadelphia, Pennsylvania 19104, United States
}

\section{Abstract}

Recent advances in microfluidic technologies have enabled production of micro-scale compound bubbles that consist of gaseous cores surrounded by a thin liquid shell, achieving control and uniformity not possible using conventional techniques. These compound bubbles have demonstrated enormous utility as functional materials for drug delivery, as ultra-lightweight structural materials, as engineered acoustic materials, and also as a separating agent for extraction of metal ions from waste fluid streams. Despite these successful demonstrations, compound bubbles have largely remained at the laboratory-scale due to the slow production rates endemic to microfluidics $(<10 \mathrm{~mL} / \mathrm{hr})$. Although parallelization approaches have enabled large-scale production of simple emulsions and bubbles, its application to the production of higher order dispersions such as compound bubbles has been limited because the optimal processing window for the production of uniform compound bubbles is relatively narrow and the required channel geometry is quite complex. In this report, we demonstrate the parallelization of multi-stage flow focusing droplet generators that produce compound ternary bubbles. We parallelize 400 multistage FFG devices, generating up to $3 \mathrm{~L}\left(\sim 10^{11}\right.$ bubbles) of monodispersed $(\mathrm{CV}<5 \%)$ compound bubbles in less than 1 hour. We show that it is critical to use multi-height channels and operate each individual generator in a flow regime that is minimally sensitive to variations in the flow rate to reliably produce uniform compound bubbles. By taking advantage of the buoyancy and the high mass transfer rate that comes from the thin shells of gas-in-oil-in-water compound bubbles which reduce the diffusion length for the metal ions and thus accelerate the extraction kinetics, we 
demonstrate the utility of mass-produced compound bubbles from the parallelized device for highly efficient extraction of $\mathrm{Nd}$ ions from a model waste stream.

\section{Graphical Abstract}

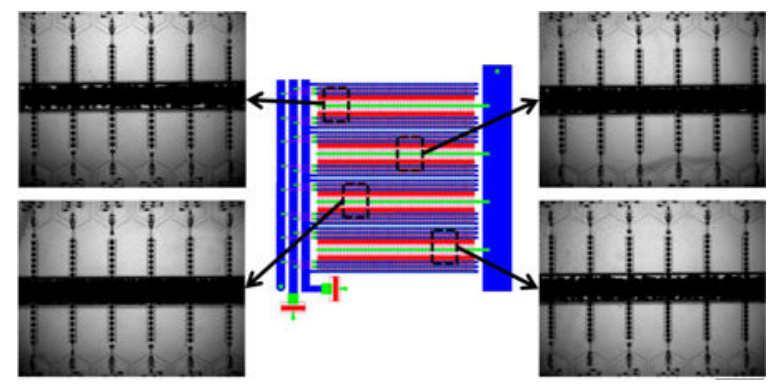

\section{Introduction}

Droplets and bubbles produced by microfluidics have advanced the fundamental understanding of multiphasic dispersions and enabled synthesis of complex particles and materials with unique morphology and useful functionality. ${ }^{1-7}$ The high uniformity of droplets and bubbles and precise control over their dimensions that microfluidics enables make them especially useful in the manufacturing of materials that are used for drug delivery, biomedical imaging, and therapeutics. ${ }^{8-11}$ Moreover these droplets can be used in high throughput screening applications to find rare molecules and cells of desired properties. 12-14 The prospect of translating these laboratory-scale demonstrations into commercial success has been further heightened by the recent advances in the large-scale production of droplets and bubbles using parallelized microfluidic generators. ${ }^{15-22}$

One important class of multiphasic fluid dispersions that microfluidics are uniquely well suited to produce are ternary compound bubbles..$^{23-25}$ Because emulsions in a microfluidic device are precisely fabricated one particle at a time, highly monodispersed multi-order emulsions and bubbles can be generated with precise control. In particular, microfluidics have been used to generate core-shell suspensions with gaseous cores and liquid shells dispersed in a continuous liquid medium such as gas-in-oil-in-water $(\mathrm{G} / \mathrm{O} / \mathrm{W})$ or gas-inwater-in-oil (G/W/O) compound bubbles. The use of microfluidics facilitates the production of these complex suspensions by enabling sequential formation of gas-in-liquid $(\mathrm{G} / \mathrm{L})$ bubbles and gas-in-liquid-in-liquid (G/L/L) compound bubbles. By controlling the geometry of the device and/or controlling the flow rates of the three fluid phases, compound bubbles of a wide range of dimension, composition and morphology can be produced. ${ }^{26-29}$ These compound bubbles have been used to produce nanoparticle-shelled bubbles for lightweight composites and polymer-shelled bubbles for acoustic applications. ${ }^{4,30-33}$

Despite these advances, potential adoption of these technologies in practical applications is hampered by low production rates associated microfluidics. Although recent advances have shown that the production of microfluidic emulsions and bubbles can be scaled-up, these developments have mostly been limited to simple emulsions and gas bubbles. It is quite challenging to optimize microfluidic devices for compound bubble generation because key 
operational parameters must be optimized to match the frequencies of bubble/drop generation in multi-stage flow focusing generators. Any mismatch in frequencies can result in non-uniform encapsulation of gas bubbles or jetting of the dispersed phase, leading to irregular break-up of oil/water phases and formation of non-uniform suspensions.

In this work, we demonstrate that compound bubbles composed of a gaseous core and a thin liquid shell can be produced at high throughput using a parallelized microfluidic device. We show that to produce uniform compound bubbles at high production rates, there are several key design considerations that must be satisfied in the development of the device architecture and in the control of the flow conditions. This work is the first of its kind to show that a parallelization strategy can be used to produce compound bubbles at rates greater than $1 \mathrm{~L} / \mathrm{hr}$. In addition to the large-scale production of $\mathrm{G} / \mathrm{L} / \mathrm{L}$ compound bubbles, we demonstrate application of $\mathrm{G} / \mathrm{L} / \mathrm{L}$ compound bubbles in the separation of rare earth elements (REEs) from waste streams. In conventional methods that mix oil and water, partitioning of the REEs into an oil phase is limited by mass transfer between the two liquid phases, which limit the efficiency and the rate of separation. By producing $\mathrm{G} / \mathrm{L} / \mathrm{L}$ compound bubbles with thin liquid shells of oil via parallelization, the rate of metal ion extraction can be significantly enhanced because of reduced diffusion lengths and the ease of isolating the oil phase due to the high buoyancy of the compound bubbles. ${ }^{34-36}$ Our demonstration of microfluidic-produced dispersion for environmental remediation and metal extraction offers a new frontier in the process intensification for extraction of sparse metal ions from aqueous phases.

\section{Experimental methods}

\section{Fabrication of microfluidic device.}

To parallelize a large number of multi-stage compound bubble generators, we fabricate a three-dimensional network to distribution the multiple phases, and collect the output from an array of compound flow focusing droplet generators. As described in our previous report, three dimensional microfluidic networks can be fabricated using a double-sided imprinting method that molds a three dimensional microfluidic chip in one-step by sandwiching PDMS between a hard silicon master and a soft PDMS master. ${ }^{19,}{ }^{21}$ These masters can be formed by conventional photo- and soft-lithography. Briefly, we prepare the triple-height hard master by photo-lithography using SU-8 3000 photoresist (MicroChem Corporation) on a silicon substrate. The first layer for FFGs is spin-coated on a silicon wafer at a thickness of $45 \mu \mathrm{m}$. After UV exposure using a mask aligner (ABM 3000HR), the second layer of $45 \mu \mathrm{m}$ thickness for backward-facing-step structure in outlet channel of FFG is directly spin-coated onto the first layer. After alignment of photomask with first layer using a mask aligner, UV exposure is performed. Finally, a third layer for intermediate holes is spin-coated at $500 \mu \mathrm{m}$ which is then irradiated with UV light. After removal of uncured photoresist, we obtain the triple-layered SU-8 patterns as a hard master. The PDMS soft master with millimeter-scale channel can be prepared by conventional single layer photolithography. Thickness of 1200 $\mu \mathrm{m}$ is formed on a silicon wafer by post baking at $95{ }^{\circ} \mathrm{C}$ for $20 \mathrm{hrs}$ and then is irradiated with UV light $(4500 \mathrm{~mJ} / \mathrm{cm})$. The final PDMS soft master is obtained by conventional softlithography using Sylgard 184 silicone elastomer (10:1 ratio of prepolymer and cross- 
linker). All SU-8 patterns and PDMS mold are silanized with tridecafluoro-1,1,2,2tetrahydrooctyl-1-trichlorosilane. The design for the 400 multi-stage FFG device (AutoCAD file) is provided in the electronic supplementary information.

Next, double-sided imprinting using the hard and soft masters is performed to fabricate the three-dimensional network in a monolithic PDMS sheet. Uncured PDMS mixture (10:1 ratio) is poured onto both soft and hard master and then degassed in a vacuum chamber. The soft and hard masters are aligned with the desired alignment patterns on both masters and then cured in a $65^{\circ} \mathrm{C}$ oven for more than four hours while applying pressure on both masters. The applied pressure ensures that all connecting features between the two masters remain open and connected. After separating the masters, the final microfluidic device is obtained by plasma bonding of thin $(\sim 1 \mathrm{~mm})$ and thick $(\sim 10 \mathrm{~mm})$ PDMS substrate with injection holes on the top and bottom of the device, respectively.

\section{Microfluidic device operation}

To generate gas-in-water-oil (G/W/O) compound bubbles in a 8-FFG device, we use nitrogen gas, $5 \mathrm{wt} \%$ polyvinyl alcohol (PVA) and $0.1 \mathrm{wt} \%$ sodium dodecyl sulfate (SDS) as aqueous phase, and hexadecane containing $2 \mathrm{wt} \%$ Span 80 as the oil phase. We initiate the process by introducing nitrogen gas using a pressure regulator and then sequentially introduce the liquid phases, first the aqueous and then the oil, using syringe pumps. To test the high production rates of compound bubbles that our 400-FFGs device is capable of, we use pressure-driven flow by applying high pressure to stainless steel pressure vessels (one gallon, Alloy Products Corp) filled with aqueous and oil phases. We sequentially introduce the nitrogen gas, aqueous, and oil by controlling the pressure regulators. The diameter of compound bubbles is measured using optical microscopy (Nikon Diaphot 300 Inverted Microscope) and analyzed using ImageJ software.

\section{Rare earth element extraction and characterization.}

For quantitative measurement of the concentration of the rare earth element used in this study (Nd), UV-Visible (UV-Vis) spectrophotometry is used (Ultrospec 2100 Pro, Amersham Biosciences). Nd(III) reacts with 8-hydroxy-7-iodo-5-quinolinesulfonic acid (Ferron) and cetyltrimethyl ammonium bromide (CTAB) forming a complex with a characteristic peak at $389 \mathrm{~nm}$ in NaAc-HAc $(\mathrm{pH}=5.00)$ buffer solution. UV-Vis absorbance at $389 \mathrm{~nm}$ is measured using $\mathrm{Nd}^{3+}$ standard solutions of known concentrations to produce a calibration curve, which is used to determine the concentration of REE in the aqueous phase upon liquid-liquid extraction. A $90 \mathrm{mg} / \mathrm{L} \mathrm{Nd}^{3+}$ solution is prepared as model wastewater, and 2-ethylhexyl phosphoric acid-2-ethylhexyl ester (P507) is used as the extractant. The oil phase comprises 1 vol\% P507 in 99 vol\% kerosene.

To implement extraction in the parallelized microfluidic devices, the outer and middle phases become the wastewater and extractant streams, respectively, and the inner phase is nitrogen. To reliably produce gas-in-oil-in-water $(\mathrm{G} / \mathrm{O} / \mathrm{W})$ compound bubbles, the surfaces of microfluidic devices are made hydrophilic via UV irradiation for 1 hour followed by oxygen plasma treatment for $5 \mathrm{~min}$. The modified hydrophilic PDMS surface is kept in contact with water to maintain its hydrophilicity. As shown in previous reports, ${ }^{37-39}$ 
hydrophilically modified PDMS retains its hydrophilicity for a long time when it is kept in contact with water. The extraction process is initiated by introducing nitrogen gas first then sequentially introducing the oil phase and finally wastewater. Once these compound bubbles flow out of the channel, the phase separation is achieved instantaneously due to the high buoyancy of the compound bubbles, and the residual concentration of $\mathrm{Nd}^{3+}$ is measured. For extraction via agitation (i.e., mixer-settler scheme), the phase ratio is kept the same as the flow rate ratio of the two phases in the microfluidic experiments. The mixture of aqueous and oil phase are agitated continuously and rapidly using a shaker (SK-O330-Pro, SCILOGEX). The rate of phase separation is much slower than compound bubbles and takes about $15 \mathrm{~min}$.

\section{Results and Discussion}

An effective strategy for achieving large-scale production of emulsions in microfluidics is parallelization. By incorporating a large number of droplet/bubble generators into an array on a single chip, prior reports have shown that sub-100 $\mu \mathrm{m}$ liquid droplets and gas bubbles can be produced at $>1 \mathrm{~L} / \mathrm{hr}$ scales. Although some limited success has been demonstrated for liquid-in-liquid-in-liquid (L/L/L) double emulsions, the number of parallel droplet generators and the droplet throughput were quite limited (less than $60 \mathrm{~mL} / \mathrm{hr}$ using a device with 15 parallel drop generators), and the demonstration was limited to all-liquid compound droplets. ${ }^{40}$ Here, we present a method to enable the liter-scale production of gas-in-water-inoil (G/W/O) compound bubbles using a device with 400 parallel G/W/O compound droplet generators. The geometry of each generator is based on a previously reported design, which takes advantage of a cross-junction flow focusing nozzle for the gas-in-water (G/W) bubble production, followed by a backward-facing-step-based flow-focusing generation of G/W/O compound emulsions. Unlike other high order compound bubble/emulsion generator designs that rely on jetting of the fluids ${ }^{41-42}$ or the use of co-axial glass capillary devices, ${ }^{33}$ this twostage geometry allows for the generation of compound bubbles via sequential formation of bubbles and droplets in the dripping regime and thus provides more control over the size and uniformity of the dispersions, facilitating parallelization. ${ }^{43}$ It is nevertheless critical to synchronize the formation of gas bubbles and droplets to form uniform compound bubbles. For the gas, water and oil phases, we use nitrogen, aqueous solutions containing $5 \mathrm{wt} \%$ polyvinyl alcohol (PVA) and $0.1 \mathrm{wt} \%$ sodium dodecyl sulfate (SDS), and hexadecane containing $2 \mathrm{wt} \%$ Span 80, respectively. To ensure that the fluid flow rates are uniform throughout the device, we use the previously reported ladder geometry, which has proven to be a robust approach to enable large-scale production of simple emulsions and bubbles. ${ }^{19,21}$ Briefly, gas, water and oil phases are introduced into the device through respective inlets and divided into 8 sets of distribution channels that run horizontally as illustrated in Figure 1. Each set of distribution channels are connected to $50 \mathrm{G} / \mathrm{W} / \mathrm{O}$ flow focusing generators (FFGs) through vertical intermediate channels. To ensure uniform fluid flows in all of the generators, we follow the previously established design criteria, $2 N_{\mathrm{f}}\left(R_{\mathrm{d}} / R_{\mathrm{f}}\right)<0.01$ (where the resistance of each channel is represented by $R=12 \mu l / w h^{3}$ where w, h, 1 and $\mu$ represent width, height and length of the channel and the viscosity of the fluid). ${ }^{40}$ This criteria states that the resistance of distribution channel $\left(R_{d}\right)$ between two adjacent FFGs should be significantly smaller than the resistance of a single FFG $\left(R_{f}\right)$. Since the flow within the FFG 
is multiphasic, we estimate the resistance of a FFG by using the dimensions of the gas injection channel (gray arrow in Figure 1B) because it has the smallest resistance compared to the water and oil channels of the FFG. To increase the resistance of each FFG, we add a serpentine resistor between the G/W FFG and G/W/O FFGs. We design these serpentine channels to have high resistance, by making the channel width smaller than that of the channel height $w<h$, resulting in a resistance $R \sim 1 / h w^{3}$. The channel dimensions we use are as follows: gas channel $w_{\mathrm{g}}=40 \mu \mathrm{m}, l_{\mathrm{g}}=1300 \mu \mathrm{m}$, and $h_{\mathrm{g}}=45 \mu \mathrm{m}$; serpentine middle channel $w_{\mathrm{m}}=40 \mu \mathrm{m}, l_{\mathrm{m}}=5000 \mu \mathrm{m}$, and $h_{\mathrm{m}}=45 \mu \mathrm{m}$; collection channel $w_{\mathrm{m}}=150 \mu \mathrm{m}, l_{\mathrm{m}}=$ $700 \mu \mathrm{m}$, and $h_{\mathrm{s}}=90 \mu \mathrm{m}$; supply channel $w_{\mathrm{s}}=2000 \mu \mathrm{m}, l_{\mathrm{s}}=5750 \mu \mathrm{m}$, and $h_{\mathrm{m}}=1200 \mu \mathrm{m}$; delivery channel $w_{\mathrm{d}}=600 \mu \mathrm{m}, l_{\mathrm{d}}=760 \mu \mathrm{m}$, and $h_{\mathrm{d}}=1200 \mu \mathrm{m}$, which satisfies the design criteria.

In addition to the design considerations outlined above, we find that the produced G/W/O compound bubbles maintains stable flow much more reliably if the outlet channel from the G/W/O FFG has a height higher than the rest of the FFG. As shown in Figure S1, a small but appreciable number of compound bubbles lose their gaseous inner cores when a single height FFG is used, likely due to the rupturing of thin aqueous layer, induced by the squeezing of the compound droplets by the channel as schematically illustrated in Figure S1. By increasing the height of the outlet channel, the compressive strain on the droplets is relieved and thus the compound bubbles maintain their integrity better. The produced G/W/O compound bubbles are subsequently flowed into four rows of collection channels; each row of collection channel is connected to 100 FFGs. The compound emulsions from the collection channels are subsequently flowed into a single outlet channel, through which the produced emulsions are collected.

A potential complication in the production of $\mathrm{G} / \mathrm{W} / \mathrm{O}$ compound bubbles is that three flow rates have to be controlled and optimized to enable their stable generation. Our previous study on the parallelization of simple gas bubble production has shown that it is helpful to use a device with a smaller number of parallel FFGs to study the effect of fluid flow rates on the size and uniformity of produced emulsions or bubbles to identify ideal flow rates that will lead to stable emulsion formation, and that these flow conditions can be used successfully in devices with greater numbers of FFGs. ${ }^{21}$ In this work, we use the same strategy to find optimal flow conditions. Specifically, we use a device with 8 parallel FFGs to study how the changes in the flow rates influence the formation of $\mathrm{G} / \mathrm{W} / \mathrm{O}$ compound bubbles and their uniformity.

In general, for a given flow rate ratio between the middle and outer phases, the uniformity as well as the size of the inner gas bubbles progressively increase as the gas pressure is increased. Interestingly, the overall size of the compound bubbles does not change significantly, resulting in the formation of thin shells at high pressures. However, increasing the gas pressure above certain limits results in the encapsulation of multiple gas bubbles and eventually to jetting of the oil phase, increasing the polydispersity of the compound bubbles (see Supporting Information). The onset of multiple bubble encapsulation and jetting occurs at a lower gas pressure as the flow rate ratio of middle and outer phases is decreased from 1:4 to $1: 10$, likely due to an increased drag force leading to jetting transition from the dripping regime. If the outer phase flow rates are controlled while maintaining the gas 
pressure and middle phase flow rate constant, we see that the dimension of the compound bubbles do not show significant changes; however, the size distributions show maxima (Figure 3). Thus to produce highly uniform compound bubbles, it is important to keep the flow rates of the outer phase low while keeping the gas pressure at an intermediate range. Using low flow rates for the outer phase also provides an advantage of producing suspensions with higher concentrations of compound bubbles.

Based on the knowledge on the influence of the flow rate of each fluid phase and their relative importance in maintaining stable formation of uniform $\mathrm{G} / \mathrm{W} / \mathrm{O}$ compound bubbles in the dripping regime, we are able to produce highly monodisperse $\mathrm{G} / \mathrm{W} / \mathrm{O}$ emulsions from the 400-FFG device as shown in Figure 4. By tuning the flow rates within the dripping regime, the dimensions of the bubbles also can be varied while maintaining high uniformity of the emulsion. The size of the inner gas bubbles can be tuned between 45 and $60 \mu \mathrm{m}$ by changing the gas pressure, which in turn changes the thickness of the oil layer of the G/W/O compound bubbles (Figure 4B). Under the optimized condition, we are able to produce up to 3 L of dispersed phase (i.e., gas + water phases) per hour while keeping the coefficient of variation in the compound bubble dimension smaller than $5 \%$. This rate of production is the highest value that has been reported to date by a factor of 50, to our best knowledge, for multiphasic compound emulsions and bubbles. A previous work with the highest reported throughput produced liquid-in-liquid-in-liquid double emulsion droplets that are larger than $100 \mu \mathrm{m} .^{44}$

To demonstrate the utility of compound bubbles produced using the parallelized device, we perform rare earth element (REE) extraction. Rare earth elements are critical materials in technologies such as microelectronics, medicine, and energy, and their demand is rapidly rising. ${ }^{45}$ Deposits of REE are typically not concentrated as minerals that can be easily mined, but rather are usually dispersed and mixed with other REEs. ${ }^{46}$ Unfortunately, REE mining and processing produce wastewater streams that contain significant quantities of metal ions that are currently wasted. Moreover, inappropriate disposal of these wastewater streams can cause undesirable environmental impact. ${ }^{47}$ Efficient recovery and separation of REE from such wastewater streams, therefore, has emerged as both an economic and environmental need. Liquid-liquid extraction, biological adsorption, as well as electric reduction have recently been developed and applied in this area to address the separation and recovery of REE from wastewater. ${ }^{48-50}$ One of the most promising approach to improve recovery of REEs from wastewater is liquid-liquid extraction, whereby highly hydrophobic extractants are used to separate REE metal ions from an aqueous source via the association of REE with the extractants in the oil phase. Separation via solvent extraction, however, often requires multistage process to attain sufficient separation due to high mass transfer resistance and low specific surface area between the two liquid phases. Prior works have shown that introducing gas phase in the oil phase is an efficient way to intensify the extraction process and increase the mass transfer efficiency by minimizing diffusion distance in thin liquid shell. ${ }^{34,36}$ It was found that the mass transfer coefficient in gas-filled droplet is increased up to 50 times compared to the simple oil-in-water dispersion without the gaseous core. In addition, by increasing the gas flow rate, the extraction efficiency could be increased over $90 \%$ under a high phase ratio of 200 . The use of microfluidics enables the formation of G/L/L compound bubbles with extremely thin liquid shells; in fact, it likely will not be 
possible to produce compound bubbles with such thin liquid shells using conventional methods. Therefore, the application of compound bubbles produced using the parallelized device in REE separation and recovery is promising.

The rate of REE extraction is compared between the conventional method of a mixer-settler and microfluidic compound bubbles. A $90 \mathrm{mg} / \mathrm{L} \mathrm{Nd}^{3+}$ solution is prepared as model "wastewater", and 2-ethylhexyl phosphoric acid-2-ethylhexyl ester (P507) is used as the extractant. The oil phase comprises 1 vol\% P507 in 99 vol\% kerosene, and the outer phase consists of $90 \mathrm{mg} / \mathrm{L} \mathrm{Nd}^{3+}$ (Figure 5(a)). To enable extraction of $\mathrm{Nd}$ ions from the aqueous phase into the oil phase and to facilitate straightforward recovery of the oil phase via buoyancy driven phase separation, gas-in-oil-in-water $(\mathrm{G} / \mathrm{O} / \mathrm{W})$ compound emulsions are produced using a 100-FFG device. Upon the formation of G/O/W compound bubbles, REEs partitions into the oil phase from the aqueous phase. To keep residence time of the G/O/W compound bubbles the same as that used in the mixer-settler based extraction (5 $\mathrm{min})$, a polytetrafluoroethylene (PTFE) tubing is connected to the outlet of microfluidic chip to keep $\mathrm{G} / \mathrm{O} / \mathrm{W}$ compound bubbles in contact with the outer aqueous phase. Due to the buoyancy of the compound bubbles, they cream and undergo phase separation spontaneously and rapidly once they are collected outside the microfluidic chip (Figure 5(b)). The concentration of residual $\mathrm{Nd}^{3+}$ ions in the aqueous phase is measured to quantify the rate of REE removal. In the case of the mixer-settler scheme, the mixture is allowed to undergo phase separation by removing agitation. The rate of creaming and phase separation is much slower (15 $\mathrm{min})$ than that observed in the G/O/W compound bubble system. The ratio of REEs and extractant is kept constant at 5:1 and the removal rates are compared.

In the mixer-settler scheme, the extraction efficiency depends on the strength of agitation in the mixer. As the agitation speed is increased from $200 \mathrm{rpm}$ to $500 \mathrm{rpm}$, extraction rate increases gradually, indicating that extraction is mass-transfer limited. Stronger agitation (i.e., higher RPM) results in the formation of smaller droplets and bigger surface-to-volume ratios, leading to faster extraction. When $\mathrm{G} / \mathrm{O} / \mathrm{W}$ compound bubbles produced from a 100FFG device, much more rapid extraction can be achieved as seen in Figure 6. Complete extraction can be achieved in less than $10 \mathrm{sec}$ due to the reduced diffusion length and the recovery of oil phase is facilitated by the low buoyancy of compound bubbles.

In this work, we have accomplished the scale-up of ternary compound bubble production using parallelized microfluidic devices. It is critical to use three structured channels and to tune the flow rates of the fluid phases to maintain the compound bubble formation in the dripping regime. We achieve liter-scale production of uniform $\mathrm{G} / \mathrm{L} / \mathrm{L}$ compound bubbles using a $100 \mathrm{FFG}$ device. Based on the large-scale production of compound bubbles, we demonstrate that the rate of rare earth element separation via liquid-liquid extraction can be accelerated compared to the conventional extraction method, due to the short diffusion length required and high buoyancy of the compound bubbles. While this study focuses on the application of microfluidic-based compound bubbles in potential applications in separation/environmental remediation space, we believe these multiphasic suspensions can be also useful in the formation of lightweight materials as well as complex bubbles/particles for acoustic imaging and drug delivery applications. 


\section{Supplementary Material}

Refer to Web version on PubMed Central for supplementary material.

\section{Acknowledgements}

This work was supported by the National Institute of Health (Grant 5 R01 EB022612-02) and NSF (CBET1604536). H.-H.J. was supported by Basic Science Research Program through the National Research Foundation of Korea (NRF) funded by the Ministry of Education (Grant 2017R1D1A1B03031526).

\section{References}

1. Teh SY, Lin R, Hung LH and Lee AP, Lab Chip, 2008, 8, 198-220. [PubMed: 18231657]

2. Shum HC, Abate AR, Lee D, Studart AR, Wang BG, Chen CH, Thiele J, Shah RK, Krummel A and Weitz DA, Macromol. Rapid Comm, 2010, 31, 108-118.

3. Lee TY, Choi TM, Shim TS, Frijns RAM and Kim SH, Lab Chip, 2016, 16, 3415-3440. [PubMed: 27470590]

4. Brugarolas T, Gianola DS, Zhang L, Campbell GM, Bassani JL, Feng G and Lee D, ACS Appl. Mater. Interfaces, 2014, 6, 11558-11572. [PubMed: 24956417]

5. Niu CC, Wang ZG, Lu GM, Krupka TM, Sun Y, You YF, Song WX, Ran HT, Li P and Zheng YY, Biomaterials, 2013, 34, 2307-2317. [PubMed: 23276658]

6. Shang LR, Cheng Y and Zhao YJ, Chem. Rev, 2017, 117, 7964-8040. [PubMed: 28537383]

7. Hou HL, Xu JH, Wang YD and Chen JN, Chin. J. Chem. Eng, 2016, 24, 79-85.

8. Deng NN, Yelleswarapu M and Huck WTS, J. Am. Chem. Soc, 2016, 138, 7584-7591. [PubMed: 27243596]

9. Fu FF, Shang LR, Zheng FY, Chen ZY, Wang H, Wang J, Gu ZZ and Zhao YJ, ACS Appl. Mater. Interfaces, 2016, 8, 13840-13848. [PubMed: 27214156]

10. Lorenz T, Bojko S, Bunjes H and Dietzel A, Lab Chip, 2018, 18, 627-638. [PubMed: 29345261]

11. Liu Y and Jiang XY, Lab Chip, 2017, 17, 3960-3978. [PubMed: 28913530]

12. Eastburn DJ, Sciambi A and Abate AR, Nucleic Acids Res, 2014, 42.

13. Baret JC, Miller OJ, Taly V, Ryckelynck M, El-Harrak A, Frenz L, Rick C, Samuels ML, Hutchison JB, Agresti JJ, Link DR, Weitz DA and Griffiths AD, Lab Chip, 2009, 9, 1850-1858. [PubMed: 19532959]

14. Tirandazi P and Hidrovo CH, Sensor. Actuat. B-Chem, 2018, 267, 279-293.

15. Nisisako T and Torii T, Lab Chip, 2008, 8, 287-293. [PubMed: 18231668]

16. van Dijke K, Veldhuis G, Schroen K and Boom R, Lab Chip, 2009, 9, 2824-2830. [PubMed: 19967120]

17. Amstad E, Chemama M, Eggersdorfer M, Arriaga LR, Brenner MP and Weitz DA, Lab Chip, 2016, 16, 4163-4172. [PubMed: 27714028]

18. Muluneh M and Issadore D, Lab Chip, 2013, 13, 4750-4754. [PubMed: 24166156]

19. Jeong HH, Yelleswarapu VR, Yadavali S, Issadore D and Lee D, Lab Chip, 2015, 15, 4387-4392. [PubMed: 26428950]

20. Yadavali S, Jeong HH, Lee D and Issadore D, Nat. Commun, 2018, 9. [PubMed: 29339724]

21. Jeong HH, Yadavali S, Issadore D and Lee D, Lab Chip, 2017, 17, 2667-2673. [PubMed: 28702573]

22. Jeong H-H, Issadore D and Lee D, Korean J. Chem. Eng, 2016, 33, 1757-1766.

23. Wan J, Bick A, Sullivan M and Stone HA, Adv. Mater, 2008, 20, 3314-3318.

24. Xu JH, Chen R, Wang YD and Luo GS, Lab Chip, 2012, 12, 2029-2036. [PubMed: 22508390]

25. Lu TY, Fan R, Delgadillo LF and Wan JD, Lab Chip, 2016, 16, 1587-1592. [PubMed: 27025654]

26. Wang WT, Chen R, Xu JH, Wang YD and Luo GS, Chem. Eng. J, 2015, 263, 412-418.

27. Kendall MR, Bardin D, Shih R, Dayton PA and Lee AP, Bubble Sci. Eng. Technol, 2012, 4, 12-20. [PubMed: 23049622] 
28. Zhang SB, Ge XH, Geng YH, Luo GS, Chen J and Xu JH, Chem. Eng. Sci, 2017, 172, 100-106.

29. Wan JD and Stone HA, Soft Matter, 2010, 6, 4677-4680.

30. Abbaspourrad A, Duncanson WJ, Lebedeva N, Kim SH, Zhushma AP, Datta SS, Dayton PA, Sheiko SS, Rubinstein M and Weitz DA, Langmuir, 2013, 29, 12352-12357. [PubMed: 24066971]

31. Duarte ARC, Unal B, Mano JF, Reis RL and Jensen KF, Langmuir, 2014, 30, 12391-12399. [PubMed: 25263163]

32. Song RY, Peng C, Xu XN, Wang JW, Yu M, Hou YM, Zou RH and Yao SH, ACS Appl. Mater. Interfaces, 2018, 10, 14312-14320. [PubMed: 29637761]

33. Lee MH, Prasad V and Lee D, Langmuir, 2010, 26, 2227-2230. [PubMed: 20039657]

34. Chen Z, Wang WT, Sang FN, Xu JH, Luo GS and Wang YD, Sep. Purif. Technol, 2017, 174, 352361.

35. Feng TF, Tan J, Deng WS and Su YF, Chem. Eng. Sci, 2018, 177, 270-283.

36. Wang WT, Sang FN, Xu JH, Wang YD and Luo GS, Rsc Adv, 2015, 5, 82056-82064.

37. Tan SH, Nguyen NT, Chua YC and Kang TG, Biomicrofluidics, 2010, 4.

38. Xu QB, Hashimoto M, Dang TT, Hoare T, Kohane DS, Whitesides GM, Langer R and Anderson DG, Small, 2009, 5, 1575-1581. [PubMed: 19296563]

39. Kim B, Perterson ETK and Papautsky I, Conf. Proc. IEEE Eng. Med. Biol. Soc, 2014, 7, $5013-$ 5016.

40. Romanowsky MB, Abate AR, Rotem A, Holtze C and Weitz DA, Lab Chip, 2012, 12, 802-807. [PubMed: 22222423]

41. Cole RH, Tran TM and Abate AR, JoVE, 2015.

42. Abate AR, Thiele J and Weitz DA, Lab Chip, 2011, 11, 253-258. [PubMed: 20967395]

43. Lee TY, Choi TM, Shim TS, Frijns RA and Kim S-H, Lab Chip, 2016, 16, 3415-3440. [PubMed: 27470590]

44. Nisisako T, Ando T and Hatsuzawa T, Lab Chip, 2012, 12, 3426-3435. [PubMed: 22806835]

45. Goodenough KM, Wall F and Merriman D, Nat. Resour. Res, 2018, 27, 201-216.

46. Hooker P, O’nions R and Pankhurst R, Chem. Geol, 1975, 16, 189-196.

47. Haque N, Hughes A, Lim S and Vernon C, Resources, 2014, 3, 614-635.

48. Hou HL, Wang YD, Xu JH and Chen JN, J. Rare Earth, 2013, 31, 1114-1118.

49. Wang X, Tu Q and Hua Z, Env. Chem, 1993, 12, 219-222.

50. Xuanxu Z, Lianping G and Danghua Y, Copper Eng, 2010, 4, 66-69. 


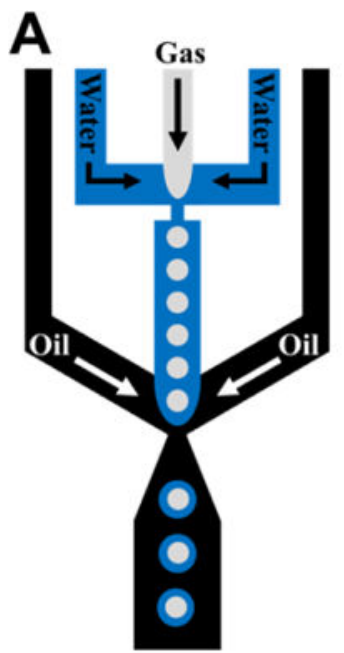

B FFGs Parallelization

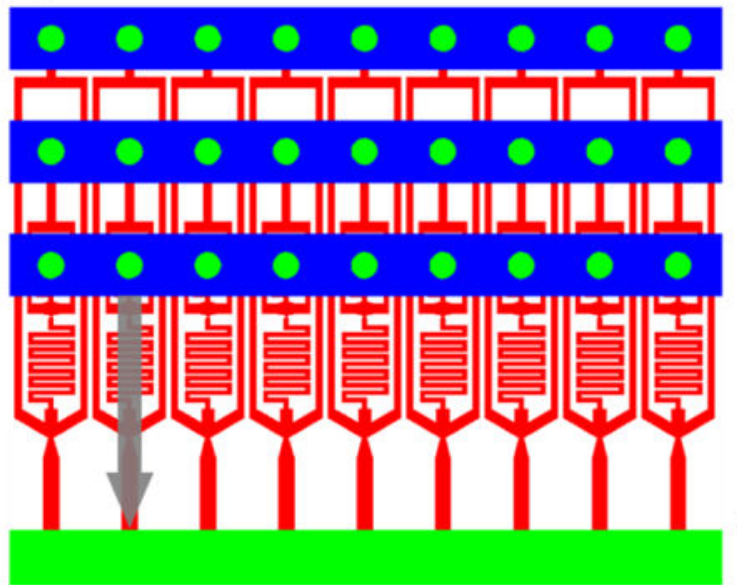

C

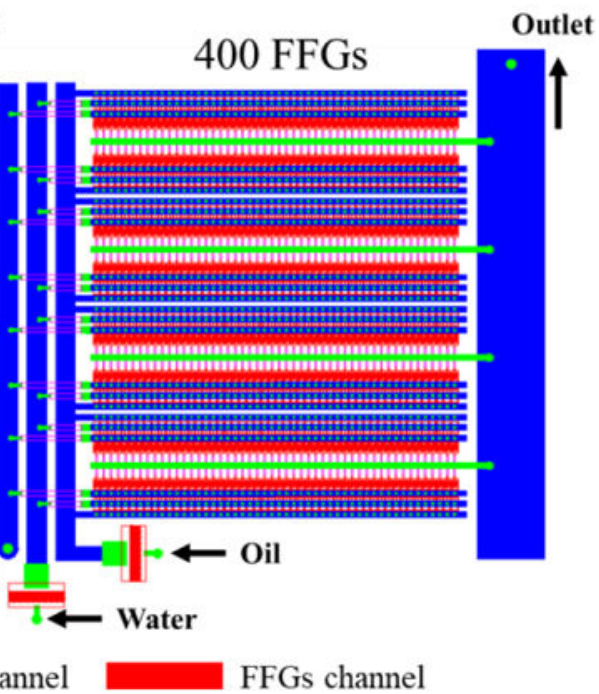

Figure 1.

Schematic illustration of (a) individual compound bubble flow focusing generator (FFG), (b) parallelized FFGs and corresponding delivery and intermediate channels, and (c) a 400-FFG parallelized microfluidic device. SEM images showing different parts of the parallel device are provided in the electronic supplementary information. 

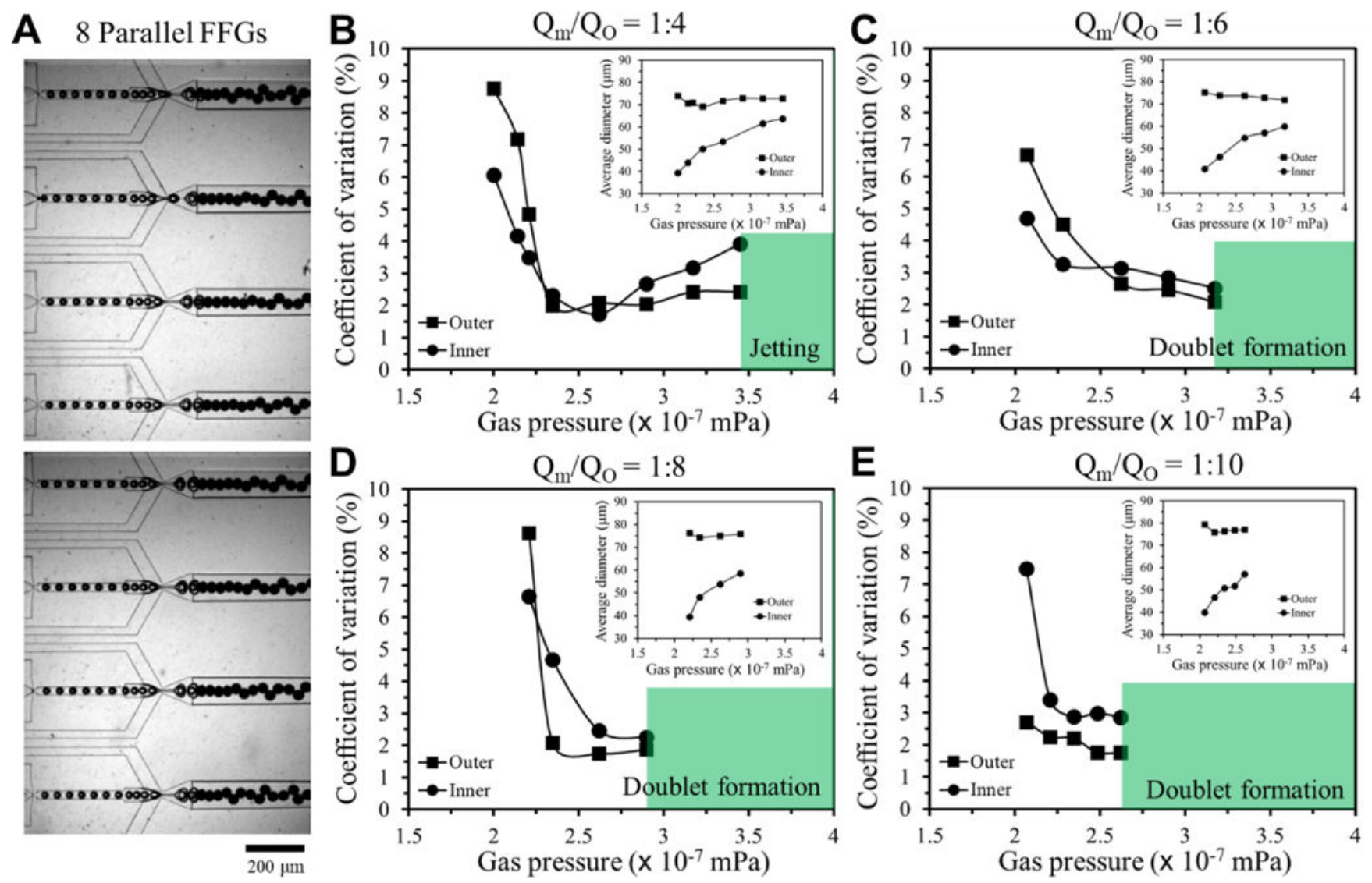

Figure 2.

(A) Optical images for generation of core-shell bubbles in 8 parallel FFG. Effect of gas pressure on the coefficient of variation and size (insets) of G/W/O compound bubbles at the middle-phase-and-outer-phase flow ratios $\left(\mathrm{Q}_{\mathrm{m}} / \mathrm{Q}_{\mathrm{o}}\right)$ of (B) 1:4, (C) 1:6, (D) 1:8 and (E) 1:10. 

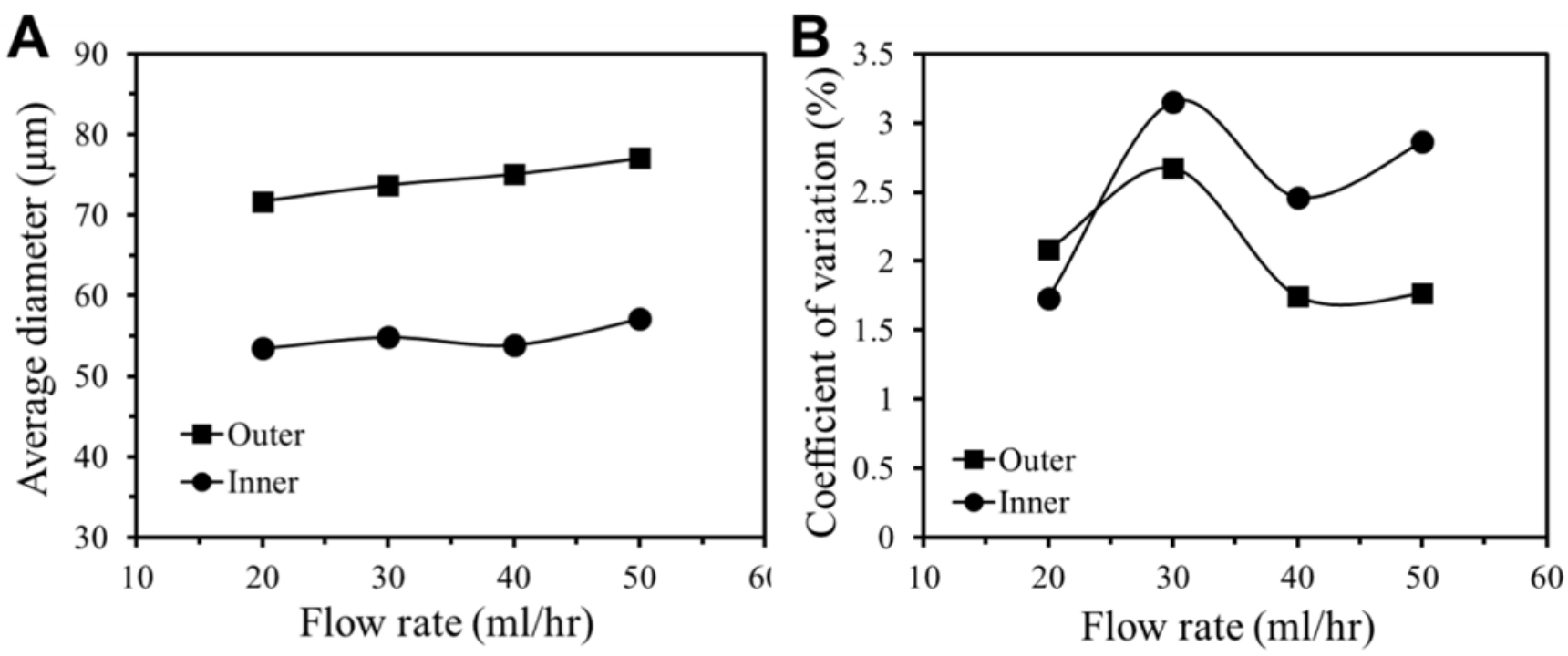

Figure 3.

Effect of outer phase flow rate on the (a) size of compound bubbles and (b) coefficient of variation. The gas pressure and the middle phase flow rate are held constant at $2.6 \mathrm{mPa}$ and $5 \mathrm{ml} / \mathrm{hr}$, respectively. 
A
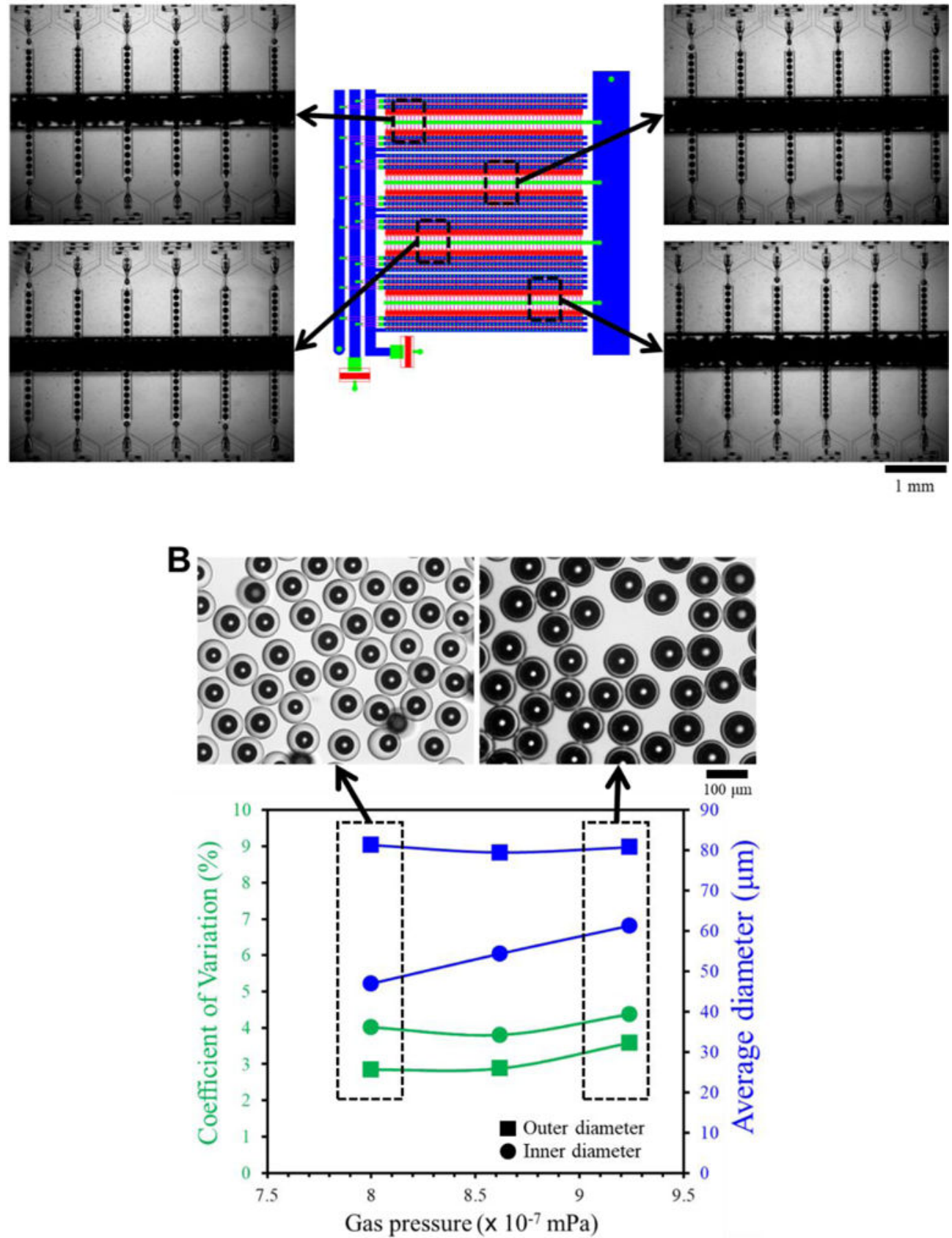

Figure 4.

Mass production of monodisperse compound bubbles in microfluidic device. (A)

Representative optical images illustrating uniform generation of $\mathrm{G} / \mathrm{W} / \mathrm{O}$ compound bubble in 400 parallelized FFGs. A movie illustrating the production of $\mathrm{G} / \mathrm{W} / \mathrm{O}$ compound bubbles is provided in the electronic supplementary information. (B) Change of compound bubble size and coefficient of variation as gas pressure generated in 400-FFGs. 


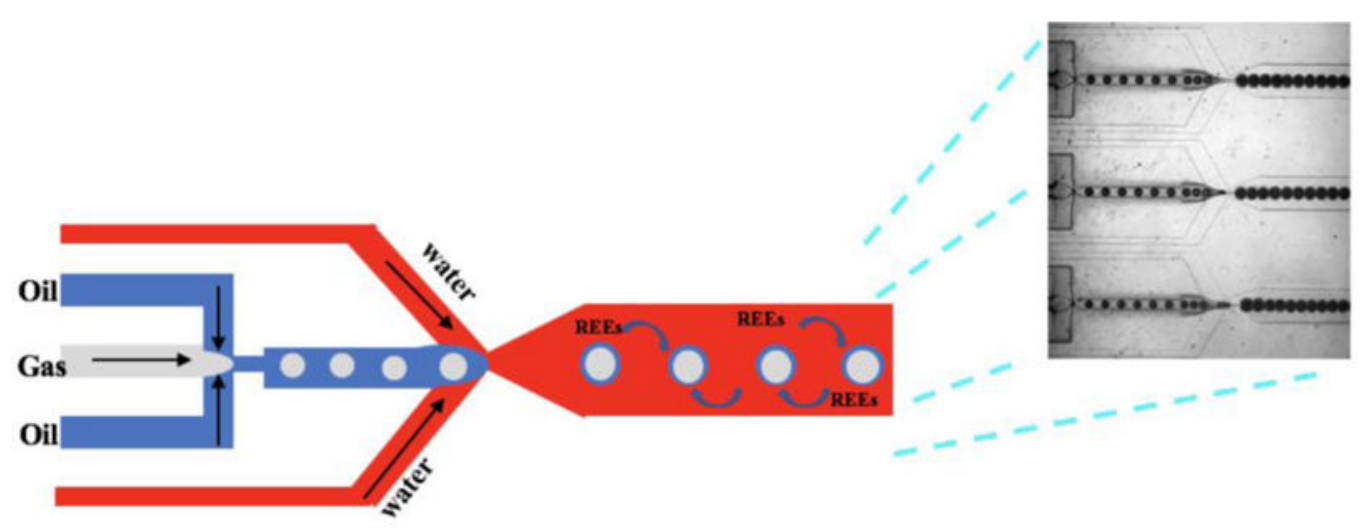

(a)

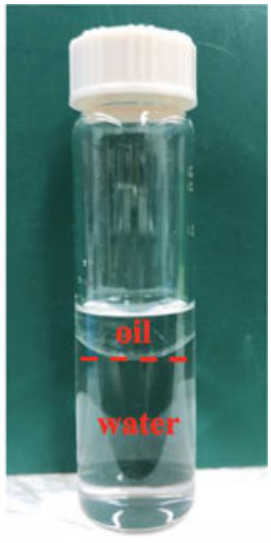

(b)

Figure 5.

(a) Schematic illustration of metal ion extraction using G/O/W compound bubbles produced using a parallelized microfluidic device. The collection channel of each compound bubble generator is $150 \mu \mathrm{m}$. (b) Phase separation of compound bubbles outside the parallelized microfluidic device. 


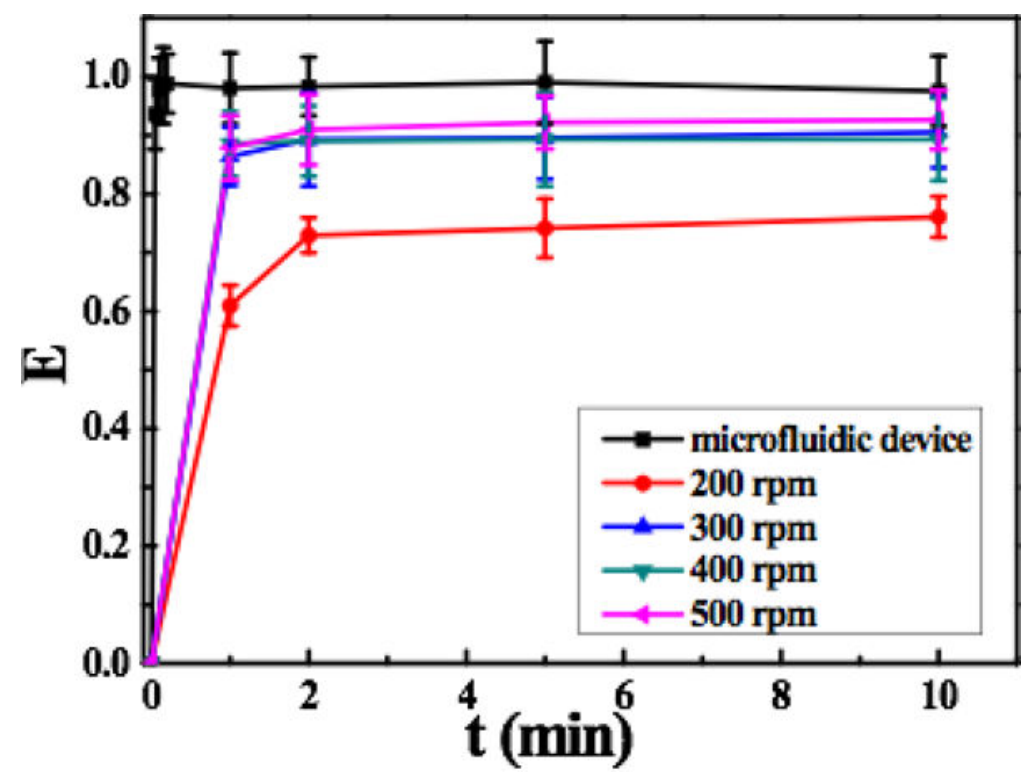

Figure 6.

Extraction efficiency (E) of $\mathrm{Nd}^{3+}$ using the $100-\mathrm{FFG} \mathrm{G} / \mathrm{O} / \mathrm{W}$ compound bubble microfluidic device and mixer-settler with different stirring speeds. 